\title{
Deep plasma etching of Parylene C patterns for biomedical applications
}

\author{
Aziliz Lecomte $^{\mathrm{a}, *}$, Aurélie Lecestre ${ }^{\mathrm{a}}$, David Bourrier ${ }^{\mathrm{a}}$, Marie-Charline Blatchéa ${ }^{\mathrm{a}}$, Laurent Jalabert ${ }^{\mathrm{a}}$, Emeline \\ Descamps $^{\mathrm{a}}$, Christian Bergaud ${ }^{\mathrm{a}}$ \\ ${ }^{a} L A A S-C N R S$, Université de Toulouse, CNRS, INSA, INP, Toulouse, France
}

\begin{abstract}
We report on the plasma etching of thick $(\sim 23 \mu \mathrm{m})$ Parylene $\mathrm{C}$ structures. Parylene $\mathrm{C}$ is a transparent polymer that benefits from high biocompatibility, flexibility and chemical inertness, and has gained increased attention over the years in the biomedical field. In the manufacturing process, highly defined structuration steps of Parylene $\mathrm{C}$ are essential, but techniques based on laser, scalpel and wet etching have shown to be unsuitable for properly cut structures. Plasma etching remains nowadays the most widespread option, though fast etching rate, lack of residues and high aspect ratios are still hard to achieve. To overcome these issues, the selection of both mask material and plasma conditions is crucial. Here, three masks -metal, positive and negative photoresists- are tested as stencils, and several plasma parameters are briefly studied in order to obtain the highest etching rate while maintaining good coverage. We showed that increasing the RF power up to a considerable $2800 \mathrm{~W}$ while maintaining a moderate physical contribution (bias power, pressure, temperature), is optimal in the achievement of fast $\mathrm{PaC}$ etching without inducing thermal stress. Besides, the addition of a short fluorinated plasma in the midst of the process is shown to alleviate residues. For the first time, negative photoresist Intervia Bump Plating (BPN) coating followed by $\mathrm{ICP}^{1}-\mathrm{RIE}^{2}$ are used in order to pattern Parylene C-based structures, with a clean cut, vertical profile and fast etching rate $(\sim 0.87 \pm 0.06 \mu \mathrm{m} / \mathrm{min})$ and a selectivity of 0.5 . This solution was carried out to release unitary Parylene-based neural probes from a silicon wafer. Finally, cytotoxicity assays on these neural implants were performed to make sure that no trace of mask or stripper residues would jeopardize device biocompatibility.
\end{abstract}

Keywords: Parylene C, plasma etching, biomedical devices, ICP-RIE, flexible neural probes

\section{Introduction}

Parylene $\mathrm{C}(\mathrm{PaC})$ is a transparent, conformably-deposited polymer that has gained attention over the years in the biomedical field. Indeed, it presents with outstanding biocompatibility (USP Class VI), excellent chemical inertness and low Young's modulus. Initially used as an encapsulation material, Parylene C is now investigated in thick layers $(\sim 20-25 \mu \mathrm{m})$ as a flexible substrate for a wide range of biomedical devices, such as retinal implants [1] or intracortical probes [2]. Therefore, there is an urgent need to master microfabrication techniques on Parylene C.

More particularly, pattern transfer of masks into Parylene C films is a critical step in Parylene microfabrication technology [3]. Parylene is chemically inert, and for this reason, chemical etching is demanding [4]. Besides, Parylene lift-off cannot be achieved because of the polymer's highly compliant nature. Finally, laser cutting or micro-abrasion might be used for coarse cutting of large samples, but is inappropriate for micro-level structuration and tends to stem debris on the openings [5]. As an alternative, plasma etching has shown great promise for the achievement of defined patterns in Parylene C films [4], or to modify topographic and chemical surface properties [6]. Because of the highly crystalline structure of this polymer, fast etching rate, lack of residues and high aspect ratios are hard to achieve, especially for thick layers [3].

In this work, the influence of both mask type and ICP-RIE plasma conditions are studied. We compare a

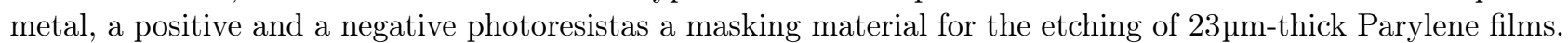
Also, the influence of several plasma parameters was assessed, such as chuck temperature, bias and ICP power,

\footnotetext{
* Corresponding author

Email address: alecomte@laas.fr (Aziliz Lecomte)

${ }^{1}$ Inductively Coupled Plasma

${ }^{2}$ Reactive Ion Etching
} 
flow and gas type. As Parylene biomedical devices are meant to interact with living things, Parylene neural probes etched out of the wafer are subject to both quantitative and qualitative viability assays in vitro.

\section{Materials and methods}

\subsection{Parylene sample preparation}

Simple 23um-thick Parylene films are coated via $\mathrm{CVD}^{3}$ on a standard 4" silicon wafer using C30S Comelec equipment. Because $\mathrm{PaC}$ has a thermal expansion coefficient 10 times higher than silicon, thermal stress can lead to PaC cracks. Therefore Parylene is pre-annealed at $150^{\circ} \mathrm{C}$ for $4 \mathrm{~h}$ under nitrogen flow to stabilize film morphology. Once the Parylene is etched through, the samples can be easily peeled off using tweezers.

After etching optimization, Parylene neural probes are also cut out of the wafer (Figure 1a). The manufacturing process for these devices is described in our previous work $[2,7]$. Briefly, $\mathrm{Ti} / \mathrm{Au}(50 / 200 \mathrm{~nm})$ is patterned via lift-off on a $21 \mu \mathrm{m}$-thick Parylene substrate. The device is then passivated with a thin $\mathrm{paC}$ layer $(\sim 1.5 \mu \mathrm{m})$, which is then thermally annealed before etched for contact openings.

\subsection{Masks and strippers}

The metal mask used is a 50nm-thick Nickel mask, patterned via lift-off over the Parylene substrate. It is removed after the plasma in a bath of $1 \% \mathrm{H}_{2} \mathrm{SO}_{4}$ mixed with $1 \% \mathrm{H}_{2} \mathrm{O}_{2}$ for a few seconds. AZ40XT
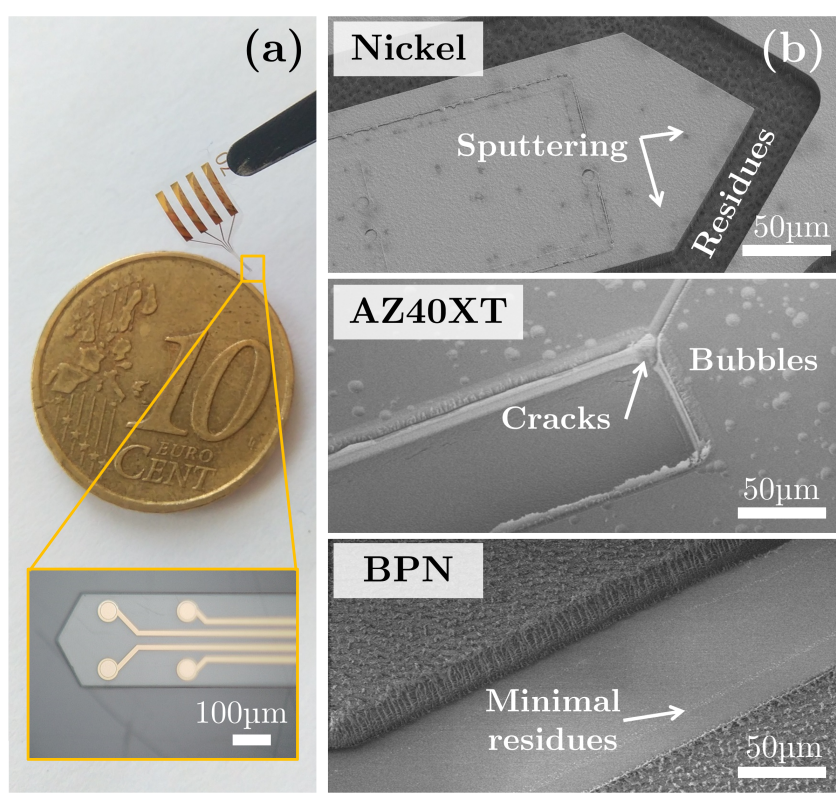

Figure 1: (a) Photograph and zoom of a Parylene-based neural probe with gold traces. (b) SEM images of plasma-etched $23 \mu \mathrm{m}-$ thick Parylene structures using different mask types. from MicroChemicals is chosen as a thick positive photoresist (PR) candidate, and can be stripped off in acetone. Finally, the negative photoresist tried out is Intervia Bump Plating Negative (BPN), a PR usually employed in electrochemistry and that can also be deposited in considerate layers. For removal, the wafer is immersed in Technics NF52 stripper for approximately 30 minutes at $80^{\circ} \mathrm{C}$, then rinsed intensively in water (10 water baths of 2 minutes).

Here, the aim is to etch trough $23 \mu \mathrm{m}$ of Parylene until reaching the silicon substrate, the etching gap dimensions and sidewalls roughness are not critical. Typical trenches etched are 50 to $100 \mu \mathrm{m}$ wide, representing $\sim 5 \%$ of the total wafer surface.

\subsection{Plasma conditions}

Two pieces of equipment are tried out for the etching of thick Parylene structures: the Trikon Omega 201 ICP-RIE ("ICP3") and the Alcatel AMS 4200 DRIE ("P4"). The parameters (flow, pressure etc.) for each test carried out with these pieces of equipment are listed in Figure 2, along with the resulting characteristics (etch rate, selectivity and ion residence time).

\subsection{Cytotoxicity assays}

The fabrication process might compromise Parylene $\mathrm{C}$ intrinsic material biocompatibility. For instance, traces of solvents such as strippers and photoresists, as well as redeposited plasma products, might stem immune reaction. To assess possible cytotoxicity, two viability assays were performed. The cell line used was SH-SY5Y, often used as in vitro models of neuronal function and differentiation.

\footnotetext{
${ }^{3}$ Chemical Vapor Deposition
} 


\begin{tabular}{|c|c|c|c|c|c|c|c|c|c|c|c|}
\hline & \multicolumn{7}{|c|}{ Parameters } & \multicolumn{4}{|c|}{ Characterization } \\
\hline & $\begin{array}{c}\text { Equip- } \\
\text { ment }\end{array}$ & $\begin{array}{l}\text { Flow } \\
(\mathrm{sccm})\end{array}$ & $\begin{array}{l}\text { Pressure } \\
\text { (mTorr) }\end{array}$ & $\begin{array}{c}\text { Temp. } \\
\left({ }^{\circ} \mathrm{C}\right)\end{array}$ & $\begin{array}{c}P_{I C P} \\
(\mathrm{~W})\end{array}$ & $\begin{array}{c}P_{\text {bias }} \\
(\mathrm{W})\end{array}$ & Gas & $\begin{array}{c}V_{P a C} \\
(\mu \mathrm{m} / \mathrm{min})\end{array}$ & $\begin{array}{c}V_{B P N} \\
(\mu \mathrm{m} / \mathrm{min})\end{array}$ & $\begin{array}{l}\text { Selectivity } \\
\left(V_{P a C} / V_{B P N}\right.\end{array}$ & $\frac{\text { Residence }}{\frac{\text { time } \tau}{(\mathrm{sec})}}$ \\
\hline (1) & ICP3 & 20 & 20 & 10 & 500 & 20 & $\mathrm{O}_{2}$ & 0.35 & 0.58 & 0.60 & 2.97 \\
\hline (2) & ICP3 & 20 & 20 & 10 & 500 & 50 & $\mathrm{O}_{2}$ & 0.40 & 0.80 & 0.50 & 2.97 \\
\hline (3) & ICP3 & 20 & 20 & 10 & 500 & 80 & $\mathrm{O}_{2}$ & 0.47 & 0.93 & 0.50 & $\overline{2.97}$ \\
\hline (4) & ICP3 & 40 & 60 & 10 & 500 & 80 & $\mathrm{O}_{2}$ & 0.40 & 0.97 & 0.41 & $\overline{4.46}$ \\
\hline (5) & ICP3 & 40 & 60 & 30 & 500 & 80 & $\mathrm{O}_{2}$ & 0.47 & 0.6 & 0.78 & $\overline{4.46}$ \\
\hline (6) & $\mathrm{P} 4$ & 200 & 40 & 10 & 2800 & 40 & $\mathrm{O}_{2}$ & 0.8 & 1.8 & 0.45 & 1.04 \\
\hline (7) & P4 & 200 & 20 & 10 & 2800 & 40 & $\begin{array}{c}\mathrm{O}_{2}+1 \min \\
\mathrm{CH}_{4} / \mathrm{C}_{4} \mathrm{~F}_{8} \\
\quad+\mathrm{O}_{2}\end{array}$ & $\begin{array}{c}\mathbf{0 . 8 7} \\
\pm 0.06^{1}\end{array}$ & $\begin{array}{c}1.53 \\
\pm 0.12^{1}\end{array}$ & $\sim 0.58$ & 0.52 \\
\hline
\end{tabular}

Figure 2: Parameters and characteristics of the plasma tests used in this study $\left({ }^{1} \mathrm{~N}=8\right)$

The MTT assay. Following the Biological evaluation of medical devices ISO 10993-5 standard, we performed a MTT cell viability assay. Parylene films tend to float, so we glue the devices using a drop of biocompatible glue (Polytec EP 601) and the glue is let dried in oven at $70^{\circ} \mathrm{C}$ overnight. Negative control wells (empty) and wells containing the devices, both in quadruplate, are sterilized in $70 \%$ ethanol and rinsed three times.

SH-SY5Y cells are cultured in DMEM with $10 \%$ fetal bovine serum, $1 \%$ L-glutamine and 1\% penicillinstreptomycin. Cells are seeded in the wells containing the Parylene probes and control wells, with approximately $10,500 \mathrm{cells} / \mathrm{cm}^{2}$. Culture plates are kept at $37^{\circ} \mathrm{C}$ with $5 \% \mathrm{CO}_{2}$. After 24,48 and $72 \mathrm{~h}$, one culture plate is retrieved, the medium is replaced by a solution of MTT (Sigma, M2128 Thiazolyl Blue Tetrazolium Bromide) at $1 \mathrm{mg} / \mathrm{ml}$ in DMEM without phenol red or serum and the plate placed back at $37^{\circ} \mathrm{C}$ during 4 hours. Then the MTT solution is removed, replaced by $\mathrm{DMSO}^{4}$ and the absorbance measured using a ThermoScientific Varioskan Flash 3001 spectrophotometer. The absorbance at $570 \mathrm{~nm}$ of the colored solution depends on mitochondrial activity of living cells, thus quantitative viability percentage can be gathered.

The fluorescent assay. We use the LIVE/DEADß Viability/ Cytotoxicity Assay Kit (Invitrogen L3224). The kit is composed of a two-color fluorescence assay, using different dyes for live (calcein AM) and dead cells (Ethidium homodimer-1), that respectively appear in green and red when excited at $490 \mathrm{~nm}$.

SH-SY5Y cells are seeded following the same procedure as for the MTT assay. Parylene probes were not glued to the bottom of their petri dish and the DMEM was without phenol red, to avoid interference with fluorescent dyes. After 24, 48 and 72 hours, the petri dish was retrieved from the oven and the DMEM medium replaced by a solution at $0.2 \mathrm{mM}$ Calcein $/ 0.2 \mathrm{mM}$ Ethidium. Twenty minutes later, the fluorescent images were taken (BX51TF microscope).

\section{Results and discussion}

\subsection{Influence of mask type}

$\mathrm{PaC}$ is highly inert therefore the etching rate is considerable slow (between 0.1 and $1 \mu \mathrm{m} / \mathrm{min}[3]$ ). The photoresist usually etches faster than the targeted material, and so the selectivity results poor. The chosen mask material must withstand long etching rates, provide conformal coverage during the time of plasma and induce minimal debris as well as most vertical sidewalls.

Metal mask. The main advantage of a nickel is that it etches very slowly with an oxygen plasma. However, a strong plasma tends to induce sputtering and micromasking (Figure 1b). This is a critical drawback, because the Parylene layer underneath is partially attacked, compromizing device functionality. Moreover, the Parylene residues found in the openings prevents the carved Parylene pieces from proper peeling, resulting in occasional tearing of the devices.

\footnotetext{
${ }^{4}$ Dimethyl sulfoxide
} 
Positive photoresist. In our previous work, positive photoresist AZ ECI 3025 was used for the etching of thin layers of Parylene [2]. To etch 23um of Parylene C, we need a PR that can be patterned in thick layers, hence the AZ40XT. But because it is based on a diazonaphthoquinone chemistry, AZ positive resist is not ideal in terms of plasma-resistance: it quickly overheats in the plasma chamber, and within minutes formed cracks and bubbles on its surface (Figure 1b).

Negative photoresist. Intervia Bump Plating Negative (BPN) is a thick, high aspect ratio negative photoresist, that has shown to withstand a 17:1 aspect ratio with conventional photolithography processes [8]. In our case, a 50 to $60 \mathrm{\mu m}$-thick BPN layer shows great promise as a mask for our ICP-RIE process (Figure 1b). Its conformal coverage is able to withstand long and high power plasma etching, with minimal residues and good profile cut.

\subsection{Influence of plasma parameters}

Our goal is the etching of $23 \mu \mathrm{m}$-thick Parylene $\mathrm{C}$ both with a high etching rate and a good selectivity regarding the etching mask, while avoiding cracks formation due to thermal stress.

The residence time is a basic process parameter involved in the reaction rate of ionization and reactive species adsorption on the surface of the sample [9]. It is proportional to process pressure, chamber volume and inversely proportional to the gas flow.

A baseline process is defined by the etching condition (1) on ICP3 machine, where the maximum available ICP power is $500 \mathrm{~W}$ (RF coil), which defines a plasma density controlling the chemical aspect of the etching. Condition (1) intends to produce a very "soft" etching defined by low gas flow, low pressure and low bias. The $\mathrm{PaC}$ etching rate is $0.35 \mu \mathrm{m} / \mathrm{min}$ with selectivity to BPN resist of 0.6 .

Increasing the bias power while keeping all other conditions constant, intends to increase the physical contribution while keeping the residence time constant (conditions (2) and (3)). The etching rate increases to $0.47 \mathrm{\mu m} / \mathrm{min}$ while the selectivity remains similar at 0.5. However a high bias induces large thermal stress.

Increasing both the flow rate and the pressure increases the residence time by a factor 1.5 (condition (4)). We found that the $\mathrm{PaC}$ etching rate decreases to $0.4 \mu \mathrm{m} / \mathrm{min}$ and the selectivity is lower (0.41). We observed that increasing the substrate temperature drastically increases BPN etching rate (condition (5)). However we observed cracks (not shown) due to poor thermalization leading to overheat.

At this point, high bias and high temperature are not suitable conditions to ensure fast etching rate and high selectivity. To explore the impact of plasma density hence the chemical contribution, higher RF source is needed as well as high flow rate (conditions (6) and (7)). Alcatel AMS 4200 ('P4') is equipped with a 3kW generator at $13.56 \mathrm{MHz}$ and a de-coupled plasma chamber, allowing a more efficient diffusion of reactive species towards the wafer. With a chamber volume larger than ICP3, 200sccm $O_{2}$ flow rate was chosen. To limit thermal stress, an intermediate bias was selected, providing less physical contribution. By reducing the pressure, the ion mean free path is larger so that the physical contribution increases leading to high etching rate for both $\mathrm{PaC}$ and BPN resist while keeping an acceptable selectivity of 0.5 .

Finally, condition $(7)$ brings the addition of a brief fluorinated plasma, with $\mathrm{He}, \mathrm{CH}_{4}$ and $\mathrm{C}_{4} \mathrm{~F}_{8}$ gases, in the middle of the oxygen plasma. During this one-minute step, $C F_{x}$ polymers are deposited on all sample surface, including on residues, which are then removed with the oxygen plasma (Figure 3).

As a conclusion, high etching rate have been obtained by selecting the highest plasma density (chemical contribution), a moderate bias (physical contribution), and a moderate ion directivity (pressure). The recipe allows fast $\mathrm{PaC}$ etching rate at $0.87 \mu \mathrm{m} / \mathrm{min}$ with a selectivity of 0.58 over BPN resist, and prevent cracks formation during the etching process.

\subsection{Cytotoxicity assessment}


The absorbance for sample and negative control wells is plotted on Figure 4a, respectively in pink and gray columns. The graph shows that the population of living cells increases over time in a similar fashion for both negative control and sample wells, meaning that the SH-SY5Y adhered and proliferated in all wells. At 24, 48 and $72 \mathrm{~h}$, the devices have shown a positive test, since the viability percentage highly exceeds $75 \%$ (between 93 and 99\%). The variability in measurement is similar for both control and sample wells, which means it is more consistent with the manual proceedings of the MTT assay (cell seeding, solution homogeneity, etc.) than with sample fabrication.

The fluorescent images are displayed on Figure $4 \mathrm{~b}$. Between 24 and 72 hours, the SH-SY5Y live cells proliferated significantly around the probe. In this case, no dead cell was present in the probe surroundings, but a few were indeed dyed by the ethidium in the well, confirming that the assay worked fine. This fluorescent viability enables us to validate once more that our implants have bio-friendly, non-cytotoxic features.

\section{Conclusion}

This work falls within a more global approach to master microfabrication techniques for pattern transfer on thick Parylene C (23um). A thick negative photoresist $(\sim 50 \mathrm{\mu m})$, the Intervia BPN, provides conformal coverage over the plasma length, with minimal residues. The addition of a short fluorinated step in the midst of an oxygen-based plasma recipe is also shown to etch oxidized residues inside the openings, resulting in a clean cut profile. The cutting of flexible $25 \mu \mathrm{m}$-thick Parylene C-based neural electrodes was carried out. Culture cell tests were performed on these devices to show that the etching process (photoresist, plasma, stripping) does not advocate cytotoxicity. Both qualitative fluorescent imaging and quantitative MTT assays proves no significant difference in cell viability between neuron-derived cells proliferating over the devices or in negative control wells. Overall, for the first time, negative photoresist (BPN) coating followed by ICP-RIE are used in order to pattern Parylene C-based structures, with a clean cut, vertical profile, fast etching rate $(\sim 0.87 \mu \mathrm{m} / \mathrm{min})$ and conservation of device biocompatibility.

\section{Acknowledgments}

This work was supported by LAAS-CNRS micro and nano technologies platform, a member of the french Renatech network. Part of this work was financed by ANR Neuromeddle 15-CE19-0006.

\section{References}

[1] Damien C. Rodger, et al. Flexible parylene-based multielectrode array technology for high-density neural stimulation and recording. Sensors and Actuators, B: Chemical, 132(2):449-460, June 2008.

[2] V. Castagnola, et al. Parylene-based flexible neural probes with PEDOT coated surface for brain stimulation and recording. In Biosensors and Bioelectronics, volume 67, pages 450-457. Elsevier, September 2014.

[3] S. Selvarasah, et al. A reusable high aspect ratio parylene-C shadow mask technology for diverse micropatterning applications. Sensors and Actuators A: Physical, 145-146:306-315, July 2008.

[4] Ellis Meng, et al. Plasma removal of Parylene C. Journal of Micromechanics and Microengineering, 18(4):045004, April 2008. 
[5] Je Min Yoo, et al. Excimer-laser deinsulation of Parylene-C coated Utah electrode array tips. Sensors and Actuators, B: Chemical, 166-167:777-786, 2012.

[6] Dimitrios Kontziampasis, et al. Effects of Ar and O2 Plasma Etching on Parylene C: Topography versus Surface Chemistry and the Impact on Cell Viability. Plasma Processes and Polymers, 13(3):324-333, 2016.

[7] Aziliz Lecomte, et al. Silk and PEG as means to stiffen a parylene probe for insertion in the brain: toward a double time-scale tool for local drug delivery. Journal of Micromechanics and Microengineering, 25(12):125003, 2015.

[8] D. Bourrier, et al. BPN a new thick negative photoresist with high aspect ratio for MEMS applications. Microsystem Technologies, 19(3):419-423, 2013.

[9] H. H. Doh et al. Gas residence time effects on plasma parameters: Comparison between Ar and C4F8. Japanese Journal of Applied Physics, Part 1: Regular Papers and Short Notes and Review Papers, 40(5 A):3419-3426, 2001. 